Journal for ImmunoTherapy of Cancer

\title{
IL-7 expands lymphocyte populations and enhances immune responses to sipuleucel-T in patients with metastatic castration-resistant prostate cancer (mCRPC)
}

\author{
Russell K Pachynski (D , , ${ }^{1}$ Chihiro Morishima, ${ }^{2}$ Russell Szmulewitz, ${ }^{3}$ \\ Lauren Harshman (1) , ${ }^{4,5}$ Leonard Appleman, ${ }^{6}$ Paul Monk, ${ }^{7}$ Rhonda L Bitting, ${ }^{8}$ \\ Omer Kucuk, ${ }^{9}$ Frederick Millard, ${ }^{10}$ John D Seigne, ${ }^{11}$ Steven P Fling, ${ }^{12}$ \\ Holden T Maecker, ${ }^{13}$ Caroline Duault (D) , ${ }^{13}$ Nirasha Ramchurren, ${ }^{12}$ Bruce Hess, ${ }^{12}$ \\ Leonard D'Amico, ${ }^{12}$ Andreanne Lacroix, ${ }^{12}$ Judith C Kaiser, ${ }^{12}$ Michel Morre, ${ }^{14}$ \\ Anne Grégoire, ${ }^{14}$ Martin Cheever, ${ }^{12}$ Evan $Y$ Yu, ${ }^{2,12}$ Lawrence Fong ${ }^{15}$
}

To cite: Pachynski RK, Morishima C, Szmulewitz R, et al. IL-7 expands lymphocyte populations and enhances immune responses to sipuleucel-T in patients with metastatic castration-resistant prostate cancer (mCRPC). Journal for ImmunoTherapy of Cancer 2021;9:e002903. doi:10.1136/jitc-2021-002903

- Additional supplemental material is published online only. To view, please visit the journal online (http://dx.doi.org/10. 1136/jitc-2021-002903).

RKP and CM contributed equally. Accepted 03 August 2021

Check for updates

(c) Author(s) (or their employer(s)) 2021. Re-use permitted under CC BY-NC. No commercial re-use. See rights and permissions. Published by BMJ.

For numbered affiliations see end of article.

\section{Correspondence to} Dr Russell K Pachynski; rkpachynski@wustl.edu

Dr Lawrence Fong; lawrence.fong@ucsf.edu

\begin{abstract}
Background Sipuleucel-T (sip-T) is a Food and Drug Administration (FDA)-approved autologous cellular immunotherapy for metastatic castration-resistant prostate cancer (mCRPC). We hypothesized that combining sip-T with interleukin (IL)-7, a homeostatic cytokine that enhances both B and T cell development and proliferation, would augment and prolong antigen-specific immune responses against both PA2024 (the immunogen for sip-T) and prostatic acid phosphatase (PAP).
\end{abstract}

Methods Fifty-four patients with mCRPC treated with sip-T were subsequently enrolled and randomized 1:1 into observation $(n=26)$ or IL-7 $(n=28)$ arms of a phase II clinical trial (NCT01881867). Recombinant human (rh) IL-7 (CYT107) was given weekly $\times 4$. Immune responses were evaluated using flow cytometry, mass cytometry (CyTOF), interferon (IFN)- $\gamma$ ELISpot, ${ }^{3} \mathrm{H}$-thymidine incorporation, and ELISA.

Results Treatment with rhlL-7 was well tolerated. For the rhlL-7-treated, but not observation group, statistically significant lymphocyte subset expansion was found, with 2.3-2.6-fold increases in CD4 +T, CD8 +T, and CD56 bright NK cells at week 6 compared with baseline. No significant differences in PA2024 or PAP-specific T cell responses measured by IFN- $\gamma$ ELISpot assay were found between rhlL-7 and observation groups. However, antigen-specific $\mathrm{T}$ cell proliferative responses and humoral $\lg \mathrm{G}$ and $\lg \mathrm{G} / \mathrm{lgM}$ responses significantly increased over time in the rhlL-7treated group only. CyTOF analyses revealed pleiotropic effects of rhlL-7 on lymphocyte subsets, including increases in CD137 and intracellular IL-2 and IFN- $\gamma$ expression. While not powered to detect clinical outcomes, we found that $31 \%$ of patients in the rhlL-7 group had prostate specific antigen (PSA) doubling times of $>6$ months, compared with $14 \%$ in the observation group. Conclusions Treatment with rhlL-7 led to a significant expansion of CD4 + and CD8+ T cells, and CD56 bright natural killer (NK) cells compared with observation after treatment with sip-T. The rhlL-7 treatment also led to improved antigen-specific humoral and T cell proliferative responses over time as well as to increased expression of activation markers and beneficial cytokines. This is the first study to evaluate the use of rhlL-7 after sip-T in patients with $\mathrm{mCRPC}$ and demonstrates encouraging results for combination approaches to augment beneficial immune responses.

\section{INTRODUCTION}

Immunotherapy represents a promising therapeutic approach for a wide range of tumor types, with numerous Food and Drug Administration (FDA) approvals in recent years. To date, the only FDA-approved immunotherapy for metastatic castrationresistant prostate cancer (mCRPC) is sipuleucel-T (sip-T), a therapeutic autologous cellular vaccine directed against prostatic acid phosphatase (PAP). Sip-T is generated by culturing patients' peripheral blood mononuclear cells (PBMC) ex vivo with a human recombinant $\mathrm{PAP} /$ granulocytemacrophage colony-stimulating factor (GMCSF) fusion protein (PA2024), resulting in activated antigen presenting cells. The activated cellular product is then adoptively transferred back into patients across three doses. ${ }^{1}$ The pivotal randomized, Phase III IMPACT trial (NCT00065442) demonstrated a significant improvement in overall survival (OS) compared with placebo in patients with mCRPC, leading to FDA approval in 2010. In IMPACT, patients with mCRPC treated with sip-T had a median OS of 25.8 months compared with 21.7 months in the placebo control. ${ }^{2}$ While sip-T has proven single agent 
activity in mCRPC, the majority of treated patients do not have clinically-demonstrable responses (ie, prostate specific antigen (PSA) or radiographic responses). Thus, strategies to improve its efficacy are needed. A subanalysis of the IMPACT trial correlated development of an immune response against PAP and/or PA2024 with improvement in OS. ${ }^{3}$ More recently, treatment with sip-T was shown to induce 'antigen spread,' or the enhancement of immune responses to a variety of tumor antigens beyond PAP and PA2024. Together, these data suggest that augmentation of the immune response may result in clinical benefit. ${ }^{4}$

Interleukin-7 (IL-7) is an endogenous homeostatic cytokine that can stimulate the proliferation and activation of $\mathrm{T}$ cells, prevent or reverse T cell anergy, ${ }^{5}$ and augment $\mathrm{T}$ cell migration from blood into lymph nodes and peripheral organs. ${ }^{6}$ Furthermore, IL-7 can also play a role in B cell functions, such as survival and activation. ${ }^{7}$ The IL-7 receptor (IL-7R), found on both naïve and effector $\mathrm{T}$ cells, is a heterodimer comprised of CD132 (the common $\gamma$-chain receptor) and CD127 (IL-7R $\alpha$ ). IL-7 is constitutively expressed and has high expression in lymphoid tissues, ${ }^{8}$ where it binds preferentially to conventional CD8 + and CD4+ T cells compared with regulatory CD4 + T cells (Tregs), which typically express no or low levels of CD127. ${ }^{10}$ Expression of the IL-7R has been associated with the persistence of long-term, tumor specific CD8 + $\mathrm{T}$ effector cells that are able to establish immunologic memory in humans. ${ }^{11}$ Overall, recombinant human IL-7 (rhIL-7) has been safe and well-tolerated in human clinical trials, with injection site reactions and mild transient constitutional symptoms being most common. ${ }^{12}{ }^{13}$ Given rhIL-7's ability to expand and enhance lymphocyte populations and their functions, we hypothesized that the addition of rhIL-7 to sip-T would result in enhanced immune responses and potentially improve clinical outcomes.

Here, we report a multicenter prospective randomized trial of sip-T, with or without the addition of a rhIL-7 (CYT107). We show a significant treatment effect of rhIL-7 on expansion of lymphocyte subsets and enhancement of immune responses, including on $\mathrm{T}$ cell proliferation and cytokine production, and on humoral responses. While the current clinical trial was not powered to evaluate clinical efficacy, our analysis revealed an improvement in PSA doubling times (PSADT) with CYT107 compared with the observation arm. Together, these data support further evaluation in a larger clinical trial.

\section{METHODS}

\section{Clinical study design and participants}

CITN12-03 was a phase II, open-label, multicenter, randomized study of the administration of CYT107 (CHO cell-produced, rhIL-7, RevImmune, France) after the completion of sip-T. Patients with asymptomatic or minimally symptomatic mCRPC were eligible to enroll after completion of standard of care sip-T. Between 2015 and 2017, 54 patients underwent informed consent and were randomized 1:1 into observation $(\mathrm{n}=26)$ or rhIL-7 $(n=28)$ arms. Prior chemotherapy for mCRPC or investigational immunotherapy was prohibited. Patients were stratified for prior abiraterone or neoadjuvant chemotherapy with or without abiraterone in order to balance arms. Patients were required to have prior orchiectomy or have ongoing luteinizing hormone-releasing hormone (LHRH) receptor agonist or antagonist treatment, with documented testosterone of $\leq 50 \mathrm{ng} / \mathrm{dL}$, Eastern Cooperative Oncology Group (ECOG) of 0-1 or Karnofsky Performance Scale (KPS) $\geq 80 \%$, adequate organ function (absolute neutrophil count $\geq 1500 / \mu \mathrm{L}$, bilirubin $<1.5 \times$ upper limit of normal (ULN), hemoglobin $\geq 10 \mathrm{~g} / \mathrm{dL}$, platelets $\geq 100,000 / \mu \mathrm{L}$, aspartate aminotransferase (AST) and alanine aminotransferase (ALT) $\leq 2.5 \times \mathrm{ULN}$, creatinine clearance $\geq 60 \mathrm{~mL} / \mathrm{min}$ by the Cockcroft-Gault), and life expectancy of at least 6 months. Evaluable disease with a positive bone scan and/or measurable disease on CT scan and/or MRI of the abdomen and pelvis was required. Concurrent treatment with systemic steroids, history of immunodeficiency disorder or clinically significant autoimmune disease, or immunosuppressive therapy within 30 days prior to enrollment were prohibited.

\section{Procedures and assessments}

After randomization, patients in the observation and IL-7 cohorts followed the same study procedures (online supplemental figure 1), except that week 2 and week 4 study visits were optional for patients assigned to observation. IL-7 (CYT107) was administered at $10 \mu \mathrm{g} /$ $\mathrm{kg}$ subcutaneously starting within 3-7 days of completion of sip-T, and continued weekly for four doses total. The first six patients randomized to receive CYT107 were followed for an additional 4 weeks prior to the study expanding past six patients, to ensure safety and tolerability of the selected CYT107 dose. Patients in the IL-7 cohort were treated for one cycle (four doses total) or until unacceptable adverse event(s), intercurrent illness preventing further administration of treatment, or disease progression.

Imaging was performed at baseline and then every 12 weeks post therapy and at time of removal from study for progressive disease. Radiographic progression-free survival was based on RECIST V.1.1 and Prostate Cancer Working Group 2 criteria. For patients with RECIST V.1.1 measurable disease, best overall response was the best response recorded from the start of the treatment until disease progression/recurrence (the smallest measurements recorded after treatment initiation were used as the reference for progressive disease). Patients were followed for 53 weeks or until death, whichever occurred first.

Complete blood counts (CBCs) with differentials and PSA levels were locally measured at CLIA-certified laboratories using longitudinally collected blood samples. Absolute lymphocyte and neutrophil counts were 
collected from CBCs, and neutrophil to lymphocyte ratios (NLRs) were calculated from these numbers and then normalized to the baseline NLR value. Although sip-T rarely leads to PSA declines $(2.6 \%$ in the IMPACT trial, ${ }^{2}$ analysis of PSADT was performed to investigate the potential impact of rhIL-7 on PSA responses. PSADTs were calculated using the MSKCC PSADT Nomogram (https://www.mskcc.org/nomograms/prostate/psa doubling_time),${ }^{14}$ with available PSA data points starting at baseline. PSA measurements were used regardless of radiographic responses; however, those collected after patients started a subsequent anticancer therapy were excluded from the PSADT calculations. The 'percent change in PSA from baseline' was calculated using week 1 as baseline and comparing subsequent PSA values at pre-specified time points (weeks 6, 11, 23, and 53) until patients were off-study or started new anti-cancer treatment.

\section{Trial end points}

The primary end point of the trial was a comparison of the magnitude of interferon (IFN) $-\gamma$ T cell ELISpot responses to PA2024 measured at week 11 in both cohorts. Secondary end points included the clinical efficacy and tolerability of sip-T plus CYT107 compared with sip-T alone, characterization of $\mathrm{T}$ cell and antibody responses to PAP and PA2024, and the effects of CYT107 on the immune competence of patients with advanced prostate cancer. The protocol was powered to detect differences in ELISpot responses between the groups (as the primary endpoint) and was not powered to discern effects on OS or other clinical parameters.

\section{Statistical analyses}

For all correlative assays, results were compared between treatment groups using two-tailed unpaired student t-tests for unequal variances, with the threshold for meeting the null hypothesis set at 0.05 . For results compared over time in the same individuals, data were analyzed using paired two-tailed t-tests for unequal variances, with the alpha set at 0.05 .

\section{PBMC isolation}

PBMC were isolated using Ficoll-Hypaque. ${ }^{15}$ Cryopreserved PBMCs were thawed (RPMI $1640+10 \%$ human serum, $+2 \mathrm{mM}$ L-glutamine, +100 units penicillin-streptomycin) $+50 \mathrm{U} / \mathrm{mL}$ Benzonase, washed and resuspended at $2 \times 10^{6}$ cells $/ \mathrm{mL}$, and rested overnight at $37^{\circ} \mathrm{C}, 5 \% \mathrm{CO}_{2}$ before use in mass cytometry (CyTOF), proliferation and ELISpot assays.

\section{Flow cytometry}

Whole blood $(100 \mu \mathrm{L})$ was transferred to BD Trucount Tubes within 30 hours of collection and labeled with a 12-color antibody panel to identify PBMC subpopulations as described. ${ }^{15}$

\section{CyTOF}

Eight subjects from each arm were analyzed using high dimensional single cell analysis by CyTOF. On thawing, each sample was split into unstimulated and stimulated wells and rested overnight. Stimulated cells were treated with PMA/ionomycin for 4 hours. Brefeldin A and monensin (both at $5 \mu \mathrm{g} / \mathrm{mL}$ per sample, Sigma-Aldrich, St Louis, Missouri) were added to both stimulated and unstimulated cells, as was anti-CD107a conjugated with $151 \mathrm{Eu}$. Then, cells were stained for surface markers and intracellular cytokines as described previously ${ }^{16}$ with the antibody panel described in online supplemental table 1. Data collected were normalized using the Nolan Lab MATLAB normalizer (https://github.com/nolanlab/ bead-normalization/releases) and then analyzed with Cytobank (www.cytobank.org).

\section{T cell proliferation assay}

Proliferation assays were performed in blinded fashion at Dendreon as described. ${ }^{3}$ Proliferation and ELISpot assays were run in parallel using the same aliquot of thawed PBMC. PBMC were plated at 100,000 cells/well and tested in triplicate with: media alone, $50 \mu \mathrm{g} / \mathrm{mL}$ PA2024, $25 \mathrm{\mu g} / \mathrm{mL}$ PAP, $1 \mathrm{ug} / \mathrm{mL}$ CEFT (Cytomegalovirus, Epstein-Barr virus, Influenza virus, and Tetanus toxin peptides; positive control), and $3 \mu \mathrm{g} / \mathrm{mL}$ PHA-L (phytohemagglutinin-L; positive control). Plates were pulsed with ${ }^{3} \mathrm{H}$-thymidine, and ${ }^{3} \mathrm{H}$-thymidine incorporation measured using a MicroBeta 2450 Scintillation Counter. Results were obtained as counts per minute and are presented as stimulation indices (SI), defined as the ratio of the test well mean divided by the media alone well mean. ${ }^{17}$

\section{T cell ELISpot}

Antigen-specific IFN- $\gamma$ release was determined using a direct 48 hours ELISpot assay qualified ${ }^{3}$ and performed at the CITN Central Lab. MISPS4W10 plates (Millipore) were coated with $15 \mu \mathrm{g} / \mathrm{mL}$ mouse anti-human IFN- $\gamma$ (MabTech clone 1-D1K) overnight at $4^{\circ} \mathrm{C}$, then blocked. PBMC were plated at 300,000 cells/well (50,000 cells/ well for PHA-L). Antigen conditions tested in triplicate included media alone, $50 \mu \mathrm{g} / \mathrm{mL}$ PA2024, $25 \mu \mathrm{g} / \mathrm{mL}$ PAP, $1 \mathrm{ug} / \mathrm{mL}$ CEFT, and PHA-L at $10 \mu \mathrm{g} / \mathrm{mL}$. After a 48 hours incubation at $37^{\circ} \mathrm{C}, 5 \% \mathrm{CO} 2$, plates were washed and biotinylated mouse anti-human IFN- $\gamma$ monoclonal antibody (clone 7-B6-1, MabTech) was added to wells. Plates were washed with PBS, developed with StrepAvidin-ALP (1:1000) and NBT/BCIP. Plates were dried at room temperature (RT) until image capture and spot counting by a CTL ELISpot reader with Immunospot software. Data are presented as 'cSPW' (backgroundsubtracted 'Corrected Spots/Well').

\section{ELISA}

ELISAs were performed in blinded fashion at Dendreon using the Agilent BioCel robotic system. Assay plates were coated with $0.5 \mu \mathrm{g} / \mathrm{mL}$ of PAP, PA2024, or tetanus 
antigens and blocked with Superblock (Thermo Scientific, Rockford, Illinois). Twofold serially diluted serum was incubated on plates for 1.5 hours, washed, then incubated with goat-anti-human HRP conjugate at 1:4000 for anti-human IgG $+\operatorname{IgM}$ or at 1:20,000 for antihuman IgG. After 1 hour at RT, plates were washed and TMB was added and absorbance read at $450 \mathrm{~nm}$. Titers $>400$ are typically considered positive in this assay, after taking background into account. ${ }^{3}$

\section{Anti-drug antibody testing}

Anti-drug antibody (ADA) testing was performed at Eurofins I ADME Bioanalyses (France) on pre-dose (week 1) and post-dose (week 11) sera from all subjects receiving CYT107.

\section{RESULTS \\ Demographics}

A total of 54 patients were enrolled, with 26 in cohort 1 (observation) and 28 in cohort 2 (rhIL-7). Overall, the two cohorts were generally well-balanced in terms of age and ethnicity (table 1). While there was an imbalance of African-American/black patients between groups, the absolute numbers were small and the vast majority of patients in each cohort were white (rhIL-7: 89.3\%, observation: $80.8 \%$ ). ECOG performance status of 1 was assessed in $7(26.9 \%)$ and $4(14.3 \%)$ patients in the observation and rhIL-7 cohorts, respectively, with the remainder of patients being ECOG 0. All patients had confirmed mCRPC, with bone metastases present in $17(73.9 \%)$ patients in the observation and $17(65.4 \%)$ patients in the rhIL-7 groups. Of note, sip-T is not recommended for patients with visceral disease, per National Comprehensive Cancer Network (NCCN) guidelines.

\section{Differences in absolute lymphocyte counts and neutrophil-to- lymphocyte ratios with rhIL-7 treatment}

Absolute lymphocyte counts, calculated from CBC, were significantly increased in rhIL-7-treated subjects at weeks 3 , 4, 6 and 11 compared with observation subjects (all $\leq 0.03$, figure 1A). Absolute neutrophil counts were not different between the groups at any time point (figure 1B). NLRs, normalized to the baseline NLR value, were calculated; a statistically significant decrease in the normalized NLR was found at week 6 in the rhIL-7 compared with the control group ( $\mathrm{p}=0.007$ ) (figure $1 \mathrm{C}$ ).

\section{rhlL-7 treatment results in significant expansion of lymphocytes}

We further investigated the role of rhIL-7 in stimulating lymphocyte expansion after sip- $\mathrm{T}$ treatment by whole blood flow cytometry. CD45 + lymphocytes were significantly expanded in the rhIL-7 $(n=22)$ compared with the observation group $(n=14)$ at week $6(p=0.007$, figure 1D). Similarly, significant increases in the absolute numbers of $\mathrm{CD} 4+\mathrm{T}(\mathrm{p}=0.007$, figure $1 \mathrm{E})$ and $\mathrm{CD} 8+\mathrm{T}(\mathrm{p}=0.001$, figure $1 \mathrm{~F}$ ) and total CD3 $+\mathrm{T}$, and $\mathrm{CD} 56^{\text {bright }} \mathrm{NK}$ cells

\begin{tabular}{|c|c|c|c|}
\hline Variable & $\begin{array}{l}\text { IL-7 } \\
(\mathrm{N}=28)\end{array}$ & $\begin{array}{l}\text { Obs } \\
(\mathrm{N}=26)\end{array}$ & $\begin{array}{l}\text { Total } \\
(\mathrm{N}=54)\end{array}$ \\
\hline \multicolumn{4}{|l|}{ Age } \\
\hline $\mathrm{N}$ & 28 & 26 & 54 \\
\hline Median & 68 & 66.5 & 67 \\
\hline Min-max & $51-81$ & $46-81$ & $46-81$ \\
\hline \multicolumn{4}{|l|}{ Race } \\
\hline White & $25(89.3 \%)$ & $21(80.8 \%)$ & $46(85.2 \%)$ \\
\hline $\begin{array}{l}\text { Black or African- } \\
\text { American }\end{array}$ & $2(7.1 \%)$ & $4(15.4 \%)$ & $6(11.1 \%)$ \\
\hline Not reported & $1(3.6 \%)$ & $1(3.8 \%)$ & $2(3.7 \%)$ \\
\hline \multicolumn{4}{|l|}{ Baseline hemoglobin } \\
\hline Median & 12.9 & 12.2 & 12.7 \\
\hline Min-max & $10.9-14.4$ & $10.3-14.7$ & \\
\hline \multicolumn{4}{|c|}{ Baseline alkaline phosphatase } \\
\hline Median (U/L) & 70.5 & 70.0 & 70.0 \\
\hline Min-max & $39-151$ & $29-1113$ & \\
\hline \multicolumn{4}{|l|}{ Baseline PSA* } \\
\hline Mean & 29.0 & 35.6 & 31.9 \\
\hline Median & 5.8 & 15.4 & 10.6 \\
\hline Min-Max & $0.11-156.4$ & $0.47-202.7$ & \\
\hline \multicolumn{4}{|l|}{ ECOG } \\
\hline 0 & $24(85.7 \%)$ & $19(73.1 \%)$ & $43(79.6 \%)$ \\
\hline 1 & $4(14.3 \%)$ & 7 (26.9\%) & $11(20.4 \%)$ \\
\hline \multicolumn{4}{|c|}{ Disease distribution $\dagger$} \\
\hline Bone only & $12(46.2 \%)$ & $14(60.9 \%)$ & $26(53.1 \%)$ \\
\hline Lymph node only & $6(23.1 \%)$ & $4(17.4 \%)$ & $10(20.4 \%)$ \\
\hline Mixed & $5(19.2 \%)$ & $3(13.0 \%)$ & $8(16.3 \%)$ \\
\hline
\end{tabular}

*For baseline PSA data, IL-7 had $n=26$, Obs had $n=21$.

†For disease distribution by imaging, IL-7 had $n=26$, Obs had $\mathrm{n}=23$.

ECOG, Eastern Cooperative Oncology Group; IL-7, interleukin-7; Obs, observation; PSA, Prostate specific antigen.

(both data not shown) were observed in the rhIL-7 group at week 6 compared with the observation group. Moreover, the rhIL-7-treated group exhibited statistically significant increases in CD4 + and CD8+ T cells at week 6 (both $\mathrm{p}<0.001$ ), and week 11 (both $\mathrm{p}<=0.004$ ) compared with baseline (figure 1E,F). However, the observation group did not exhibit such changes over time. Of the PBMC subpopulations identified by our flow cytometry panel, fold-increases were greatest at week 6 among CD8 + T cells (2.6-fold), CD4 + T cells (2.3-fold), CD56 ${ }^{\text {br }} \mathrm{NK}$ cells (2.3-fold), and B cells (1.8-fold) (figure 1G). By contrast, the observation group did not exhibit such increases (figure 1H).

\section{High-dimensional CyTOF analysis}

To further understand the impact of rhIL-7 on major PBMC subpopulations after sip-T, we performed CyTOF analysis on eight patients from both arms. This subset 
A
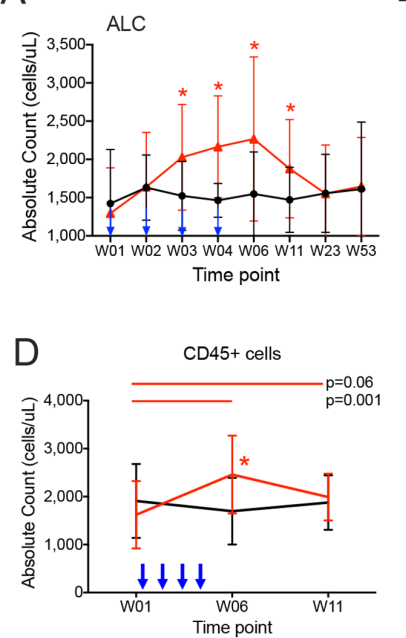

G

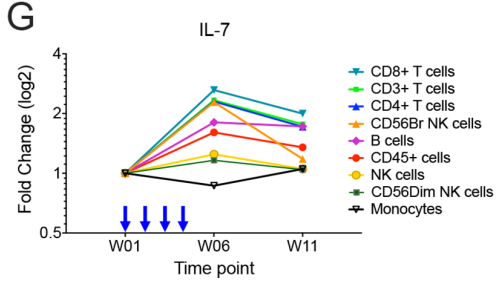

B

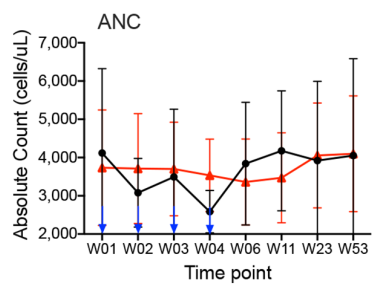

E

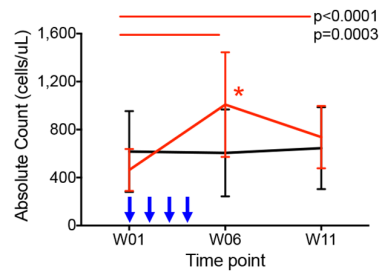

C

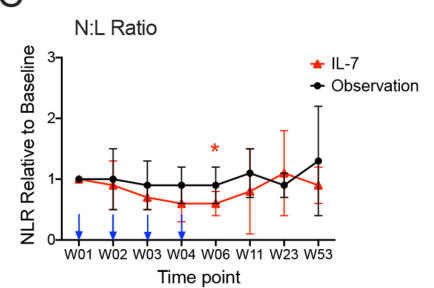

$\mathrm{F}$
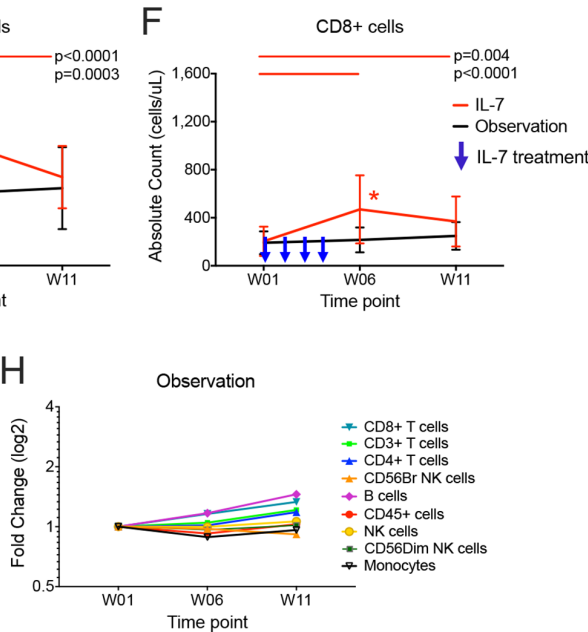

Figure 1 Lymphocyte expansion among interleukin (IL)-7-treated and observation cohorts. Absolute cell counts (cells/ $\mu$ l blood) were calculated from local complete blood count data to determine (A) absolute lymphocyte counts (ALC), (B) absolute neutrophil counts (ANC), and (C) neutrophil:lymphocyte ratios (NLR) relative to the baseline NLR. Mean and SD are shown for the IL-7-treated (red) and observation (black) cohorts. Blue arrows indicate the weekly dosing schedule for recombinant human (rh)lL-7. Asterisks indicate statistically significant differences in cell counts between the cohorts (for ALC, $p=0.005$ at week 3 , $p=0.02$ at week $4, p=0.009$ at week 6 and $p=0.03$ at week 11 ; for NLR relative to baseline, $p=0.007$ at week 6 ; all t-tests for unequal variances). Lymphocyte subset expansion was monitored using flow cytometry as described at weeks 1,6 and 11 (D-H). Mean and SD are shown for the rhIL-7-treated (red) and observation cohorts (black, (D-F). Asterisks indicate statistically significant differences in cell counts between the cohorts (at week 6: for CD45 + cells, $p=0.007$; for CD4 + cells, $p=0.007$; for CD8 + cells, $p=0.001$; all t-tests for unequal variances). Bars at the top of each plot indicate statistically significant differences between time points for the IL-7-treated cohort (red). No statistically significant differences between time points was found for the observation cohort. Log fold-changes from baseline for specific PBMC subsets is shown for the rhIL-7-treated (G) and observation $(\mathrm{H})$ cohorts.

of subjects was chosen based on strength of individual antigen-specific responses seen by ELISpot (online supplemental figure 2) and availability of adequate numbers of PBMC. The flow cytometric gating strategy for these experiments is shown in online supplemental figure 3).

To evaluate T cell functionality and anergy, the expression of immune checkpoint and costimulatory molecules was analyzed. In the rhIL-7-treated group, a clear decrease of PD-1 (programmed cell death protein 1) expression was observed at week 6 compared with baseline on CD4+, $\mathrm{CD} 8+$ and $\gamma \delta \mathrm{T}$ cells (figure 2B). Decrease in surface expression of another inhibitory molecule, CTLA-4, occurred on CD4 + T cells at week 6 in both the rhIL-7 and observation groups, and in the $\gamma \delta \mathrm{T}$ cells in the observation group only (figure 2A). We also detected a higher expression of PD-L1 (programmed cell death ligand 1) on CD4 + T, CD8 + T and NK cells, which might be due to the decrease in PD1 (figure 2C). Finally, CD137 (4-1BB) expression also increased on CD4 + and CD8+ T cells and slightly on NK cells at week 6 in the rhIL-7 treated group but not the observation group. The same trend was observed on $\gamma \delta \mathrm{T}$ cells in the rhIL-7 treated group but it did not reach statistical difference (figure 2D). Altogether, these data support the hypothesis that rhIL-7 treatment potentiates the sip- $\mathrm{T}$ therapy by increasing $\mathrm{T}$ and NK cell functionality and costimulatory potential, as well as diminishing $\mathrm{T}$ cell anergy markers.

To further investigate $\mathrm{T}$ cell functionality, we analyzed pro-inflammatory intracellular cytokine expression with or without PMA/ionomycin stimulation among T cells, NK cells, and $\gamma \delta \mathrm{T}$ cells (Figure 2 and online supplemental table 1). Increases in intracellular cytokines by week 6 were found commonly among CD $4+\mathrm{T}$ cells, $\gamma \delta \mathrm{T}$ cells and CD56 + NK cells in the rhIL-7-treated cohort (figure 2). However, statistically significant increase in intracellular cytokine levels were seen rarely in the observation cohort. Stimulated CD $8+$ and $\gamma \delta \mathrm{T}$ cells already expressed high amounts of tumour necrosis factor alpha (TNF $\alpha)$ independently of the rhIL-7 treatment so the effect of rhIL-7 treatment on these populations was not evaluable (figure 2G). Interestingly, IL-6 expression in CD4 + T 


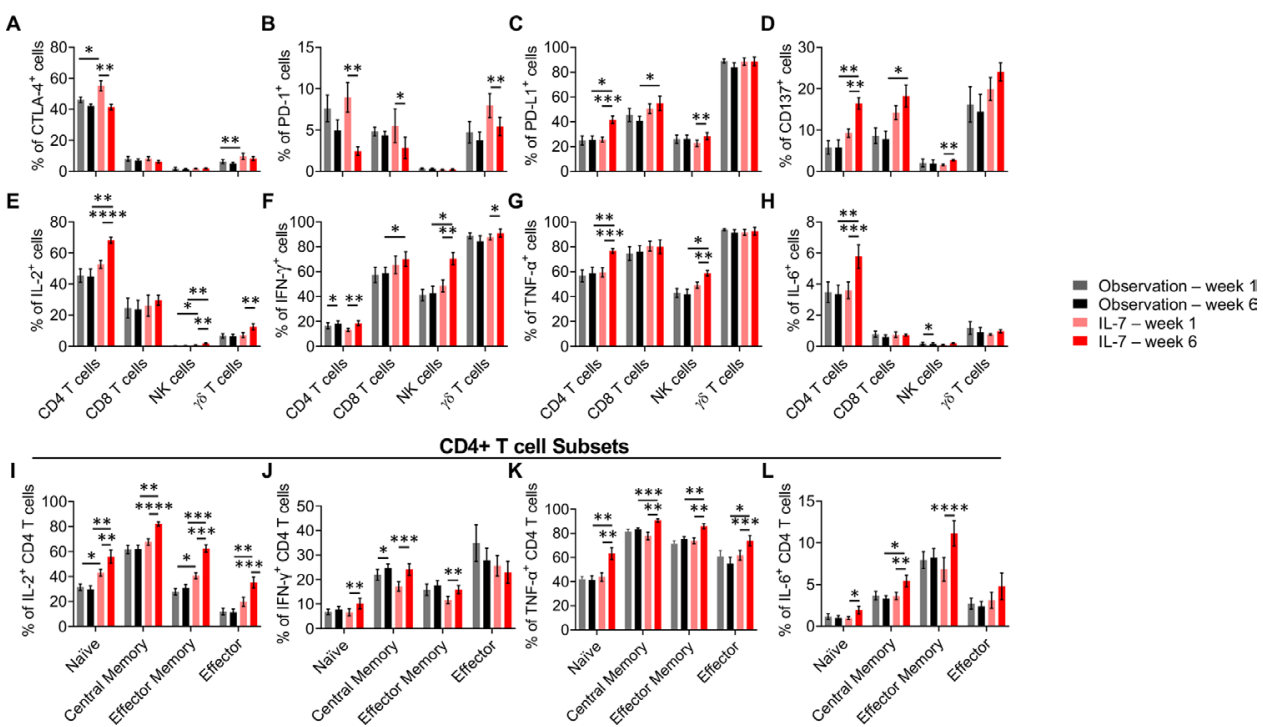

Figure 2 CyTOF analyses. CYT107 induced an increase of pro-inflammatory cytokine expression by different lymphocyte subsets. (A-C) Immune checkpoint expression and (D) costimulatory factor CD137 expression on T and NK cells were assessed by the CyTOF analysis at baseline (W01) and week 6 (W06) following PMA/lonomycin stimulation for 4 hours. PD-1 expression (B) decreased while PD-L1 and CD137 expression (C,D) increased on T cells on treatment by CYT107. (E-H) IL-2, IFN- $\gamma$, TNF- $\alpha$ and IL-6 intracellular expression in NK and T cells were analyzed by CyTOF in a subset of nine patients in both arms at baseline (W01) and week 6 post-treatment (W06) after stimulation by PMA/lonomycin for 4 hours. (I-L) Intracellular cytokine expression in CD4 T cells memory subsets. On stimulation by PMA/lonomycin, IL-2 and TNF- $\alpha$ expressions $(\mathrm{I}, \mathrm{K})$ were upregulated among all naïve and memory subsets following treatment with CYT107 while IFN- $\gamma$ and IL-6 $(\mathrm{J}, \mathrm{L})$ were not increased in the terminally differentiated effector cells. Histograms represent the mean+SEM. Multiple comparisons were made using mixed-effects model or two-way ANOVA. ${ }^{*} p<0.05,{ }^{* *} p<0.01,{ }^{* * *} p<0.001$. CyTOF, mass cytometry; IFN, interferon; IL, interleukin; NK, natural killer; TNF, tumor necrosis factor; PD-1, programmed cell death protein 1; PD-L1, programmed cell death ligand 1; ANOVA, analysis of variance.

cells was increased with stimulation in the rhIL-7 group at week 6 compared with baseline; although typically expressed by stromal or antigen presenting cells, IL-6 expression has been shown in $\mathrm{CD} 4+\mathrm{T}$ cells ${ }^{18}$ and may play a role in the mobilization and antitumor activity of NK cells ${ }^{19}$ (figure $2 \mathrm{H}$ ). As CD4 + T cells were particularly sensitive to rhIL-7 potentiation, we investigated whether rhIL-7 treatment targeted a specific CD $4+$ memory T cell subset (figure 2I-L). Surprisingly, we detected an increase of IL-2 and TNF $\alpha$ expression across all the naive and memory subsets in the rhIL-7 arm at week 6 (figure 2I and $\mathrm{K}$ ), while IFN- $\gamma$ and IL- 6 were upregulated in the naïve, central memory and effector memory subsets (figure 2J and L), demonstrating that rhIL-7 appears to broadly stimulate the entire CD4 + T cell compartment the most, among $\mathrm{CD} 4+, \mathrm{CD} 8+, \gamma \delta+\mathrm{T}$ cells and NK cells. Potentially relevant to the IFN- $\gamma$ ELISpot results, fewer CD $4+$ T cells demonstrated intracellular IFN- $\gamma$-positivity than for IL-2, IL-6 or TNF- $\alpha$.

\section{T cell IFN- $\gamma$ and proliferative responses}

A major hypothesis and the primary end point of this clinical trial was that treatment with rhIL-7 would enhance T cell IFN- $\gamma$ responses to PA2024 by week 11 compared with observation. However, no significant increase in $\mathrm{T}$ cell IFN- $\gamma$ response to PA2024, PAP, or CEFT was detected in the rhIL-7 group $(n=28)$ compared with the observation group $(\mathrm{n}=24)$ at week 11 (online supplemental figure 2).
Furthermore, no significant increases in IFN- $\gamma$ responses to any of the antigens over time was found for either group. However, it is important to note that while the percentage of antigen-specific responses as measured in the ELISpot assay-which uses a fixed number of PBMC/ well-was not different between the two groups, the $\sim 2$ to 3-fold increases in total body lymphocytes (figure 1A) and 2-fold increases in CD4 and CD8 T cells (figure 1G) seen in the IL-7 arm would-in effect-translate into overall higher total absolute numbers of antigen-specific effector $\mathrm{T}$ cells in the body.

Antigen-specific $\mathrm{T}$ cell proliferative responses against PA2024, PAP, and CEFT were also measured (figure 3). It is worth noting that background counts incorporated in the absence of antigen were unusually high in both groups at baseline, with similar results at later time points. Since all of the samples demonstrated high levels of background, no results were excluded on the basis of this characteristic. Despite the high bar required to overcome this background proliferation, likely due to prior sip-T treatment, robust antigen-specific proliferative responses (SI), particularly to PA2024, were identified. No statistically significant differences were observed between treatment arms at any time point, but proliferative responses to PA2024 and PAP increased significantly over time in the rhIL-7-treated group in contrast to CEFT responses. Only PA2024-specific proliferative responses increased 
IL-7
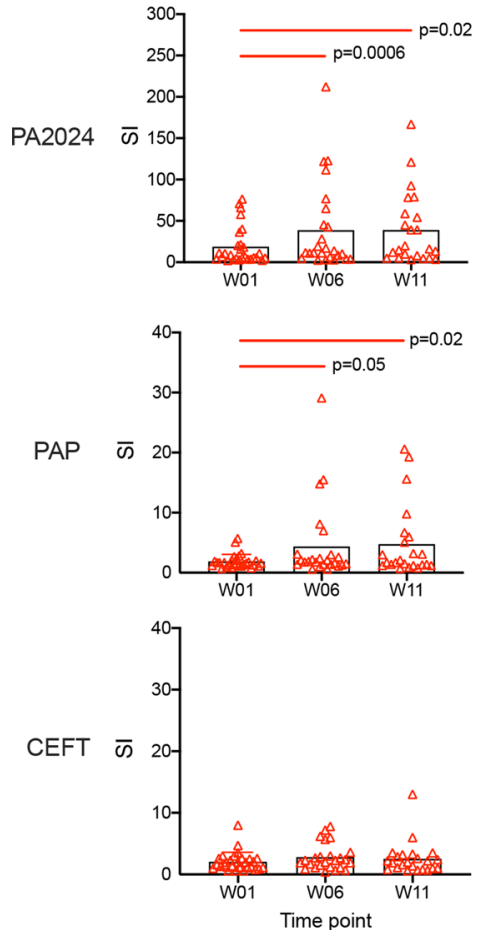

Observation
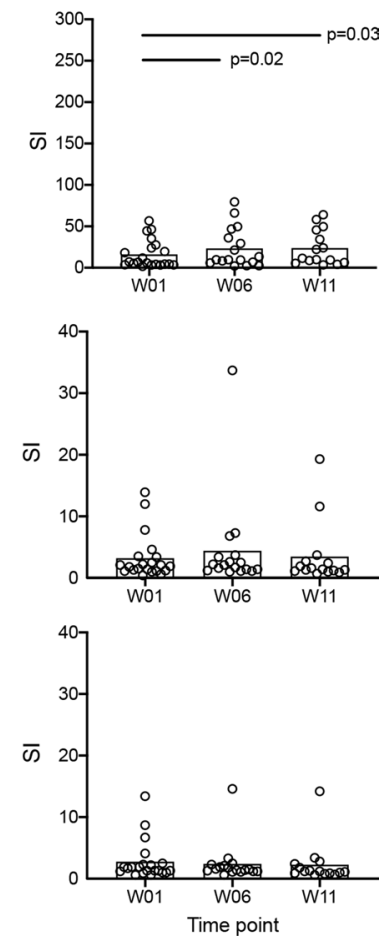

Figure 3 Proliferative responses to PA2024 and PAP increase over time. Each symbol represents data from an individual patient in either IL-7-treated (left panels, red) or observation (right panels, black) arms obtained at baseline (week 1, W01), and weeks 6 (W06) and 11 (W11). Mean stimulation index for each antigen tested (PA2024, PAP, and CEFT) at each time point is indicated by the black columns. Bars at the top of each plot indicate statistically significant differences between time points for the recombinant human IL-7-treated (red) and observation cohorts (black). IL, interleukin; PAP, prostatic acid phosphatase; SI, stimulation indices; CEFT, CMV/EBV/infleunza/tetanus control peptides.

significantly over time in the observation group. Proliferative responses to PAP were less robust than to PA2024 at all time points $(\mathrm{p}<0.0001$ for all $)$. However, PAP responses increased significantly over time in the rhIL-7-treated group ( $\mathrm{p}=0.05$ at week $6, \mathrm{p}=0.02$ at week 11 ) but not in the observation group (figure 3 ).

\section{Antigen-specific humoral responses with rhIL-7 treatment}

As IL-7 has been shown to play roles in B cell activation and function, ${ }^{7}$ we examined the impact of rhIL-7 administration on antibody responses. IgG alone and IgG plus IgM antibody responses to PA2024 and PAP were measured at baseline and weeks 6 and 11. The antibody titers shown in figure 4 represent the greatest serum dilution yielding a positive result. As all patients had previously been treated with sip-T, it was not surprizing that positive PA2024specific IgG responses were detected at baseline in $16 \%$ $(4 / 25)$ and $30 \%(6 / 20)$ of rhIL-7-treated and observation group patients, respectively, before rhIL-7 treatment. Positive baseline PAP IgG responses were also detected in $30 \%(8 / 27)$ and $25 \%$ (5/20) of rhIL-7-treated and observation group patients, respectively. Even higher numbers

IL-7

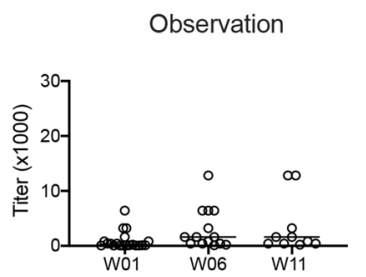

PA2024 IgG
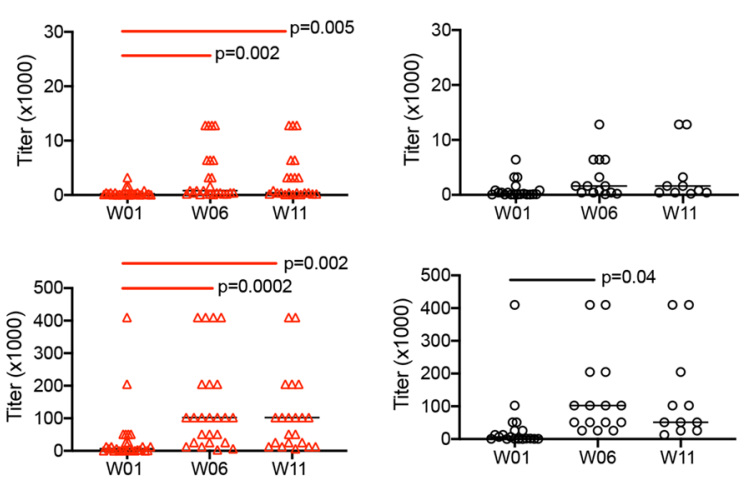

PA2024 $\lg \mathrm{G} / \mathrm{lgM}$
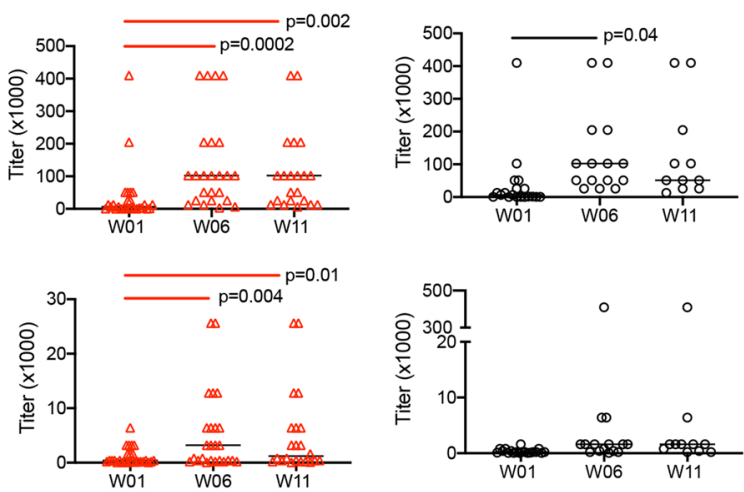

PAP

$\lg \mathrm{G} / \lg M$
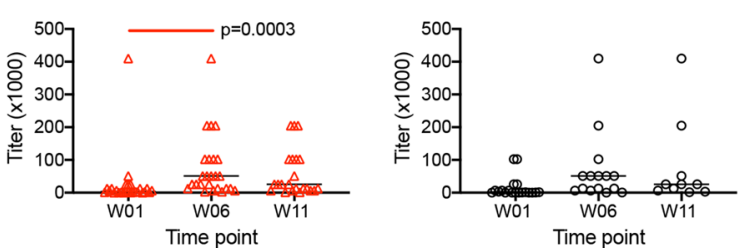

Figure 4 Antibody responses to both PA2024 and PAP increase over time in rhlL-7-treated patients. Each symbol represents data from an individual patient in either rhIL-7treated (left panels, red) or observation (right panels, black) arms obtained at baseline (week 1, W01), and weeks 6 (W06) and 11 (W11). Both IgG and IgG plus IgM responses against PA2024 and PAP antigens were evaluated using serially diluted sera and established positivity thresholds as described. ${ }^{3}$ Mean antibody titers at each time point are indicated by the black lines. Bars at the top of each plot indicate statistically significant differences between time points for the rhIL-7-treated (red) and observation cohorts (black). IL, interleukin; PAP, prostatic acid phosphatase; rhIL7 , recombinant human IL-7.

of patients had positive $\mathrm{IgG} / \mathrm{IgM}$ responses to PA2024 $(85 \%(23 / 27)$ in rhIL-7\% and $90 \%(17 / 19)$ in observation groups) and PAP (89\% (23/26) in rhIL-7\% and $63 \%$ $(12 / 19)$ in observation groups) at baseline. These data are consistent with previous studies showing induction of humoral PA2024 and PAP-specific immune responses with sip-T therapy. ${ }^{3}$ No statistically significant differences were found between the rhIL-7-treated and observation cohorts at any time point. However, despite the presence of positive antigen-specific antibody responses at baseline, IgG and IgG/IgM titers to both PAP and PA2024 significantly increased by week 6 in the rhIL-7 group. By contrast, only the PA2024 IgG/IgM response was significantly increased at week 6 in the observation group.

\section{Adverse effects with rhlL-7 treatment after sip-T}

The safety, tolerability, and related adverse events (AE) were collected in both the observation and rhIL-7 groups over the course of the trial and assessed using the 
National Cancer Institute Common Terminology Criteria for Adverse Events V.4.0. After the completion of sip-T, patients in the observation arm generally tolerated it well, with $6 / 26(23.1 \%$ ) having any grade related AEs and 0/26 $(0 \%)$ having any related grade $3 / 4$ AEs (online supplemental table 2). Overall, the rhIL-7 cohort had higher rates of treatment-related AEs, with 26/29 (92.9\%) having any $\mathrm{AE}$, and 2/28 (7.1\%) having grade 3 or greater AEs. Injection site reactions, all grades 1-2 in 21 of 28 patients, comprised the majority of the AEs in the rhIL-7 group. The two grade $3+$ AEs were hypertension and diarrhea. Fatigue in $7 / 28(25 \%)$, skin disorders in $7 / 28$ (25\%), pruritus/urticaria in 3/28 (10.7\%) each, and arthralgias in $3 / 28(10.7 \%)$, were the next most common grade $1-2$ AEs. Overall, the addition of rhIL-7 following sip-T was well tolerated with no treatment-related grade 5 AEs, and no unexpected toxicity compared with prior studies. Only 1 of 28 subjects tested positive for ADA in confirmatory testing but was not tested for neutralizing antibodies.

\section{Clinical efficacy of rhlL-7 treatment after sip-T}

A secondary objective of the study was to assess clinical outcomes. While insufficient data were available to determine statistically powered differences, we saw no significant differences in radiographic progression free survival (rPFS) or in OS between the two groups (online supplemental figure 4). Intriguingly, the IL-7 group does show tails of the curves in both OS and rPFS, with median OS not yet reached in the IL-7 group. Importantly, however, the study was not adequately powered to determine differences in these or other clinical outcomes and long-term follow-up was completed on December 31, 2018; thus these findings can only be hypothesis-generating.

Treatment with sip-T typically does not result in robust PSA responses, with only $2.6 \%$ of patients in the IMPACT trial having a $>50 \%$ PSA response (PSA50). ${ }^{2}$ Regardless, we also analyzed PSA levels for potential treatment effects. Baseline PSA measurements between groups were not statistically different (figure $5 \mathrm{~A}$ ), nor were any significant differences in mean PSAs found between groups at any time point. We calculated the per cent change in PSA from baseline at pre-specified time points (weeks 6, 11, 23, and 53). For the rhIL-7-treated group, a statistically significant decrease in the PSA per cent change from baseline was found only at week 6 compared with the observation group $(\mathrm{p}=0.042$, figure $5 \mathrm{C})$. Notably, PSADT of $>6$ months, calculated using the validated MSKCC nomogram, ${ }^{14}$ were found to be more than twice as common in the IL-7-treated group compared with the observation group ( $31 \%$ vs $14 \%$ ) (figure $5 \mathrm{~B}$ ). While not sufficiently powered to detect such PSA differences, we found higher rates of both PSA50 $(4.3 \%$ vs $0 \%)$ and any negative per cent change in PSA (30.4\% vs $10.5 \%)$ at week 6 in the rhIL-7-treated group compared with the observation group.

The two rhIL-7-treated subjects (numbers 51 and 34) with exceptional PSADT responses were diagnosed with lymph node only involvement in the abdomen or pelvis,
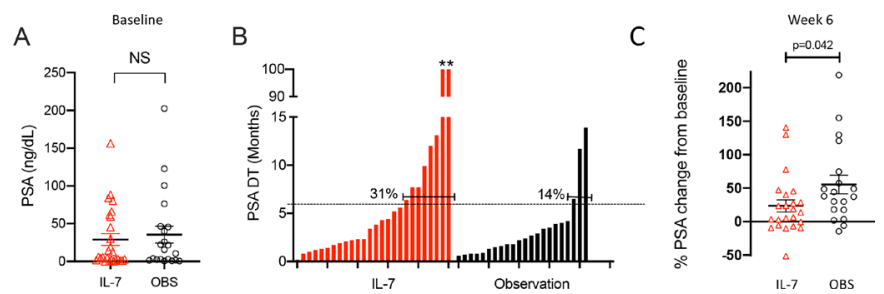

Figure 5 PSA data. (A) Baseline PSA levels are shown for each patient in the IL-7-treated (red) and observation (black) cohorts. (B) PSA doubling time (PSADT, in months) was calculated using the Memorial Sloan-Kettering calculator (https://www.mskcc.org/nomograms/prostate/psa_doubling_ time). Each bar represents data from a single patient; PSA values obtained after patients started another anticancer therapy were not used for calculations. Two patients in the IL-7-treated cohort had negative PSADT values calculated due to persistent reductions in their PSA values; these were arbitrarily assigned PSADT of 100 months as previously described, ${ }^{29}$ and are indicated by asterisks. The percentage of patients with a PSADT above the threshold of 6 months are shown. (C) Per cent change in PSA levels from baseline to week 6 in individual patients (IL-7, $n=23$; observation, $n=19$ ). IL-7, interleukin-7; OBS, observation.

had alkaline phosphatase levels within the normal range, and stable disease as a best radiographic response. They were among 16 of $28(57 \%)$ of rhIL-7 treated patients with stable disease; 13 of $26(50 \%)$ subjects in the observation group also had stable disease. Only one subject in the rhIL-7 group had a partial response. It is worth noting that $5 / 28(18 \%)$ and $10 / 26(38 \%)$ subjects in the rhIL-7 and observation groups, respectively, did not have response measurements available. Of these two subjects, subject 51 had very robust immune responses by nearly all assays tested; PA2024 and PAP-specific proliferation increased 3.6-fold (80th percentile) and 2.1-fold (76th percentile), respectively, by week 6 , while PA2024-specific IgG levels also increased 2.6-fold (83rd percentile) by week 6 among rhIL-7-treated patients. Both subjects had relatively robust $\mathrm{CD} 4+\mathrm{T}$ cell expansion at 2.4-fold and 2.5-fold increases at week 6 , while subject 51 also had a substantial B cell expansion of 2.8-fold. In addition, both had NLR decreases of $60 \%$ by week 6 which were at the 75th (subject 34) and 83rd (subject 51) percentiles. Notably, both demonstrated robust IFN- $\gamma$ ELISpot responses to PA2024 and PAP at both weeks 6 and 11 . Subject 34 had PA2024-specific increases of 2.3-fold (76th percentile) and ninefold (92nd percentile) at weeks 6 and 11, respectively, while subject 51 had 1.6-fold (64th percentile) and 4.1-fold (79th percentile) at weeks 6 and 11, respectively. Finally, subject 34 had PAP-specific IFN- $\gamma$ ELISpot increases of 61.2-fold (100th percentile) and 63.2-fold (86th percentile) at weeks 6 and 11, respectively, while subject 51 had increases of sevenfold (76th percentile) and 2.8-fold (71st percentile) at weeks 6 and 11 , respectively. With respect to the OS of these exceptional PSA responders, we have limited data as protocolspecified follow-up was completed at week 53; however, both of these patients were alive at last follow-up. Taken 
together, these data suggest rhIL-7 treatment may alter prostate cancer disease kinetics and related immune parameters.

\section{DISCUSSION}

Here, we present the first study to evaluate the use of rhIL-7 in patients with mCRPC. Patients were enrolled and randomized after receiving sip-T, a treatment known to induce both cellular and humoral immune responses, including antigen-specific $\mathrm{T}$ cell responses. Given the beneficial effects of rhIL-7 on lymphocyte expansion and function, we hypothesized that rhIL-7 treatment immediately following standard of care sip-T would augment immune responses, and potentially clinical responses, compared with the sip-T-treated observation group. While the primary endpoint of a statistically significant difference in PA2024-specific T cell IFN- $\gamma$ ELISpot responses was not met, significant increases in antigen-specific T cell proliferative and humoral responses were induced by rhIL-7 over the course of treatment. Moreover, these significantly enhanced $\mathrm{T}$ and $\mathrm{B}$ cell responses targeted the PAP antigen in addition to PA2024. Not surprisingly, treatment with rhIL-7 resulted in a significant expansion of total CD45+, CD4+, and CD8 + cells at week 6 compared with observation. Finally, encouraging clinical findings were seen in the rhIL-7-treated group compared with the observation group, with decreased PSA values at week 6 and an increase in the number of patients with PSADTs of $>6$ months.

Our data confirm that administration of rhIL-7 can induce significant in vivo increases in lymphocyte subsets, including CD4 + and CD8+ T cells, leading to a statistically significant decrease in NLR at week 6 . The total absolute increase in lymphocyte populations seen with rhIL-7 treatment also suggests that, while the 'frequency within PBMC' of antigen specific T cells was not increased (as measured by ELISpot), the total pool (ie, total body lymphocytes) of antigen-specific effector $\mathrm{T}$ cells was increased (along with other T cells). This is because the ELISpot assay only measured specific $\mathrm{T}$ cells within a fixed number of PBMC, while flow cytometry and ALC measure absolute numbers. In-depth mechanistic CyTOF analyses on stimulated PBMC from a subset of patients also revealed significant increases in beneficial "proinflammatory' cytokines (IL-2, IFN- $\gamma$, TNF- $\alpha$ and IL-6) in the rhIL-7-treated group, particularly among CD4 + T cells.

Multiple factors likely contributed to our inability to reach the primary end point. First, the study was intended to enroll 40 patients per arm in order to identify a twofold difference in IFN- $\gamma$ ELISpot responses between the groups. Due to unexpected limitations on the availability of rhIL-7 during the trial, only 54 total patients were enrolled (with 52 samples evaluated) out of the planned 80 , limiting our statistical power to discern differences between the groups. Patient retention in the observation arm over time, in the absence of a placebo, was also a challenge. Another possible confounder is the observation that preceding sip- $\mathrm{T}$ therapy had initiated effects on both $\mathrm{T}$ and $\mathrm{B}$ cell responses, leading to higher 'background' levels of immune stimulation detected in both groups prior to randomization to observation or treatment with rhIL-7. These 'baseline' immune responses, measured after standard-of-care therapy with sip-T, effectively 'raised the bar' even higher for the ability to detect differences between the two groups, as we did not have access to patient samples or data prior to treatment with sip-T. Alternatively or in addition, the activated state of immune cells at baseline documented by higher expression levels of CTLA-4 and PD-1 could have made T cells more refractory to subsequent stimulation by rhIL-7 than if they had not been previously stimulated by sip-T. Finally, the CyTOF analyses demonstrated that even with mitogen stimulation, intracellular T cell IFN- $\gamma$ levels were modest and similar between rhIL-7 and observation groups, consistent with our inability to detect statistically significant changes in this parameter between groups using the antigen-stimulated ELISpot assay.

Clinically, we confirmed that rhIL-7 treatment was generally well tolerated, with expected injection site and skin reactions, and an acceptable safety profile in line with prior studies. We did not expect to detect meaningful efficacy changes with the addition of rhIL-7 to sip-T, as the study was not powered to detect these. Unexpectedly, however, we observed a decrease in the PSA change from baseline in the rhIL-7 arm at week 6 , and an increase in the number of patients having PSADTs of $>6$ months, compared with observation. Given that baseline PSAs were not significantly different between groups, this favorable change in PSADTs could suggest a clinical impact of the significant lymphocyte expansion in the rhIL-7 group, but this hypothesis will have to be formally explored in larger, adequately powered trials.

Our study had several weaknesses. We only tested immune responses in peripheral blood, limiting our ability to identify direct relationships between rhIL-7 administration and antitumor responses in the tumor microenvironment. As mentioned, early termination of enrollment led to a smaller than expected trial size, and thus reduced the power of our analyses. There were also a greater number of African-Americans (AA) in the observation group $(n=4)$ compared with the IL-7 $(n=2)$ group. As AA patients with mCRPC may have improved responses to sip- $\mathrm{T}^{20}$, this imbalance may have contributed to the outcomes; regardless, we were still able to show significant differences in immune responses, week 6 PSA responses, as well as improved PSADTs in the IL-7 group. While patients were maintained on ADT, they were allowed to be treated with additional FDA-approved agents or enroll in additional clinical trials during follow-up after completion of sip-T and subsequent rhIL-7 or observation. Thus, these subsequent therapies could have altered clinical responses seen; importantly, PSA data collected from patients after starting subsequent anticancer therapies was not included in PSA or PSADT calculations. Clinical 
follow-up was stopped at week 53 per protocol, which also limited our ability to determine any potential impact of IL-7 on long-term OS in these patients, which has been seen in other mCRPC immunotherapy studies. ${ }^{21}$

To date, sip-T remains the only FDA-approved immunotherapy for unselected patients with MCRPC and the search for additional immunotherapeutics for patients with prostate cancer continues. Single agent checkpoint inhibitor trials of ipilimumab, nivolumab, and pembrolizumab have been disappointing in mCRPC. For example, single agent pembrolizumab showed an ORR of $5 \%$ in PD-L1 positive patients, ${ }^{22}$ while single agent atezolizumab produced a PSA response in only $8.6 \%$ of patients. ${ }^{23}$ Another large phase III trial of a single agent immunotherapy, the PSA-based vaccine Prostvac-VF, was also recently reported as negative when compared with placebo in chemotherapy-naive patients with mCRPC. ${ }^{24}$ The failure of such single agent trials supports the rationale and need for future combinatorial approaches such as ours. Other combination approaches are currently being studied using various immunotherapeutic agents in mCRPC, including combination ipilimumab and nivolumab, ${ }^{25}$ PAP-encoding DNA vaccine in combination with pembrolizumab, ${ }^{26}$ attenuated listeria vaccine targeting PSA in combination with pembrolizumab, ${ }^{27}$ and other innovative multi-immunotherapy adaptive approaches. $^{28}$

While our data clearly demonstrated rhIL-7's effects on $\mathrm{CD} 4+$ and CD8 + T cell expansion when given after si-T, the limitations of our study impaired our ability to find associations between the immune findings and clinical outcomes. The finding that greater numbers of CD4 + $\mathrm{T}$ cells capable of producing pro-inflammatory cytokines were detected in rhIL-7-treated individuals raises the question of whether sequencing of rhIL-7 before or during sip- $\mathrm{T}$ treatment may produce more naive $\mathrm{T}$ cells for sip-T stimulation, ultimately leading to improved clinical responses. It is also interesting to consider whether patients with lower (or higher) baseline ALC might incur more clinical benefit from rhIL-7. Overall, our data indicate an acceptable safety profile and immunological rationale for using rhIL-7 in combination with sip-T as well as other immunostimulatory agents, such as checkpoint inhibitors and targeted radiotherapeutics, together. We believe that additional combination studies such as these are needed to improve outcomes for patients with mCRPC.

In summary, while sip-T remains a backbone of prostate cancer immunotherapy, clinical studies to date have made it clear that single agent immunotherapies, such as checkpoint inhibitors, are not efficacious in mCRPC. Thus, combination therapies must be pursued. A strength of this clinical trial was the cooperative, multi-institutional, randomized nature of the study. It is also the first to evaluate the addition of a rhIL-7 product in patients receiving sip-T. rhIL-7 treatment was safe and well tolerated. Among rhIL-7-treated patients, we demonstrated a significant expansion in lymphocyte populations, a significant reversal of the NLR, and significant increases in antigenspecific T and B cell responses, including to PAP. Findings from these studies support further exploration of rhIL-7 as part of a combination immunotherapy approach.

\section{Author affiliations}

${ }^{1}$ Department of Medicine, Washington University School of Medicine, St Louis, M0, USA

${ }^{2}$ University of Washington, Seattle, Washington, USA

${ }^{3}$ University of Chicago Pritzker School of Medicine, Chicago, Illinois, USA

${ }^{4}$ Medical Oncology, Dana Farber Cancer Institute, Boston, Massachusetts, USA

${ }^{5 *}$ Current affiliation: Surface Oncology, Cambridge, MA, USA

${ }^{6}$ UPMC, Pittsburgh, Pennsylvania, USA

${ }^{7}$ Ohio State University James Cancer Hospital, Columbus, Ohio, USA

${ }^{8}$ Wake Forest Baptist Health, Winston-Salem, North Carolina, USA

${ }^{9}$ Department of Hematology and Medical Oncology, Emory University School of

Medicine, Atlanta, Georgia, USA

${ }^{10}$ University of California San Diego, La Jolla, California, USA

${ }^{11}$ Dartmouth-Hitchcock Medical Center, Lebanon, New Hampshire, USA

${ }^{12}$ Fred Hutchinson Cancer Research Center, Seattle, Washington, USA

${ }^{13}$ Institute for Immunity, Transplantation and Infection, Stanford University, Stanford, CA, USA

${ }^{14}$ Revlmmune, Paris, France

${ }^{15}$ University of California San Francisco, San Francisco, California, USA

Twitter Russell K Pachynski @RPachynski, Lauren Harshman @LaurenCHarshman and Judith C Kaiser @Fluffone

Acknowledgements We are deeply indebted to the patients and their families and to staff at all clinical sites for their participation in and support of this study. This work was supported by grants from the National Cancer Institute: U01CA154967 and UM1CA154967 (to MC and SPF), Cancer Center Support Grant P30 CA015704 (to $\mathrm{MC}$ ), and by Dendreon (Seattle, Washington, USA). In addition, support was provided by Revimmune (Paris, France). HTM and CD are supported by grant 1U24CA224309 from the NIH. LH is currently an employee of Surface Oncology, Cambridge, Massachusetts, USA.

Contributors RKP: investigation, data analysis, writing —original draft, review and editing. CM: investigation, methodology, data analysis, writing — original draft, review and editing. RS: investigation, writing —original draft, review and editing. LH: investigation, writing —review and editing. LA: investigation, writing —review and editing. PM: investigation, writing — review and editing. RLB: investigation, writing — review and editing. OK: investigation, writing —review and editing. FM: investigation, writing — review and editing. JDS: investigation, writing —review and editing. SPF: investigation, methodology, data analysis, writing-original draft, review and editing, supervision, project administration, funding acquisition. HTM: investigation, data analysis, writing - original draft, review and editing. $\mathrm{CD}$ : investigation, data analysis, writing —original draft, review and editing. NR: investigation, data analysis, writing — review and editing. BH: investigation, data analysis, writing — review and editing. LD: investigation, data analysis, writing - review and editing. AL: project administration, supervision, data curation, writing - review and editing. JCK: project administration, supervision, data curation, writing — review and editing. MM: supervision, funding acquisition, writing — review and editing. AG: supervision, funding acquisition, writing —review and editing. MC: conceptualization, methodology, data analysis, writing — review and editing, supervision, project administration, funding acquisition. EYY: conceptualization, investigation, methodology, writing — review and editing. LF: conceptualization, investigation, methodology, data analysis, writing —original draft, review and editing, supervision, project administration, funding acquisition.

Funding This work was supported by grants from the National Cancer Institute and additional funding from Dendreon (Seattle, Washington) and support from Revlmmune (Paris, France).

Competing interests $M M$ and $A G$ are employees of Revlmmune who provided CYT107 for this trial. LF, RKP and PM served in advisory/consulting roles for Dendreon. EYY has received grant funding from Dendreon.

Patient consent for publication Not required.

Ethics approval All patients signed Institutional Review Board-approved informed consents.

Provenance and peer review Not commissioned; externally peer reviewed. 
Data availability statement Data are available upon reasonable request. All data relevant to the study are included in the article or uploaded as supplementary information.

Supplemental material This content has been supplied by the author(s). It has not been vetted by BMJ Publishing Group Limited (BMJ) and may not have been peer-reviewed. Any opinions or recommendations discussed are solely those of the author(s) and are not endorsed by BMJ. BMJ disclaims all liability and responsibility arising from any reliance placed on the content. Where the content includes any translated material, BMJ does not warrant the accuracy and reliability of the translations (including but not limited to local regulations, clinical guidelines, terminology, drug names and drug dosages), and is not responsible for any error and/or omissions arising from translation and adaptation or otherwise.

Open access This is an open access article distributed in accordance with the Creative Commons Attribution Non Commercial (CC BY-NC 4.0) license, which permits others to distribute, remix, adapt, build upon this work non-commercially, and license their derivative works on different terms, provided the original work is properly cited, appropriate credit is given, any changes made indicated, and the use is non-commercial. See http://creativecommons.org/licenses/by-nc/4.0/.

\section{ORCID iDs}

Russell K Pachynski http://orcid.org/0000-0002-8966-7631

Lauren Harshman http://orcid.org/0000-0002-7636-1588

Caroline Duault http://orcid.org/0000-0003-2742-1668

\section{REFERENCES}

1 Cheever MA, Higano CS. Provenge (Sipuleucel-T) in prostate cancer the first FDA-approved therapeutic cancer vaccine. Clin Cancer Res 2011;17:3520-6.

2 Kantoff PW, Higano CS, Shore ND, et al. Sipuleucel-T immunotherapy for castration-resistant prostate cancer. N Engl J Med 2010;363:411-22.

3 Sheikh NA, Petrylak D, Kantoff PW, et al. Sipuleucel-T immune parameters correlate with survival: an analysis of the randomized phase 3 clinical trials in men with castration-resistant prostate cancer. Cancer Immunol Immunother 2013;62:137-47.

4 GuhaThakurta D, Sheikh NA, Fan L-Q, et al. Humoral immune response against nontargeted tumor antigens after treatment with Sipuleucel-T and its association with improved clinical outcome. Clin Cancer Res 2015;21:3619-30.

5 Mackall CL, Fry TJ, Gress RE. Harnessing the biology of IL-7 for therapeutic application. Nat Rev Immunol 2011;11:330-42.

6 Beq S, Rozlan S, Gautier D, et al. Injection of glycosylated recombinant simian $\mathrm{IL}-7$ provokes rapid and massive T-cell homing in rhesus macaques. Blood 2009;114:816-25.

7 Vazquez MI, Catalan-Dibene J, Zlotnik A. B cells responses and cytokine production are regulated by their immune microenvironment. Cytokine 2015;74:318-26.

8 Takada K, Jameson SC. Naive T cell homeostasis: from awareness of space to a sense of place. Nat Rev Immunol 2009;9:823-32.

9 Liu S, Riley J, Rosenberg S, et al. Comparison of common gammachain cytokines, interleukin-2, interleukin-7, and interleukin-15 for the in vitro generation of human tumor-reactive $\mathrm{T}$ lymphocytes for adoptive cell transfer therapy. J Immunother 2006;29:284-93.

10 Rodríguez-Perea AL, Arcia ED, Rueda CM, et al. Phenotypical characterization of regulatory T cells in humans and rodents. Clin Exp Immunol 2016;185:281-91.
11 Chandran SS, Paria BC, Srivastava AK, et al. Tumor-Specific effector CD8+ $T$ cells that can establish immunological memory in humans after adoptive transfer are marked by expression of IL7 receptor and c-myc. Cancer Res 2015;75:3216-26.

12 Sportès C, Babb RR, Krumlauf MC, et al. Phase I study of recombinant human interleukin-7 administration in subjects with refractory malignancy. Clin Cancer Res 2010;16:727-35.

13 Sheikh V, Porter BO, DerSimonian R, et al. Administration of interleukin-7 increases CD4 T cells in idiopathic CD4 lymphocytopenia. Blood 2016;127:977-88.

14 Pound CR, Partin AW, Eisenberger MA, et al. Natural history of progression after PSA elevation following radical prostatectomy. JAMA 1999;281:1591-7.

15 Bhardwaj N, Friedlander PA, Pavlick AC, et al. Flt3 ligand augments immune responses to anti-DEC-205-NY-ESO-1 vaccine through expansion of dendritic cell subsets. Nat Cancer 2020;1:1204-17.

16 Sahaf B, Rahman A, Maecker HT, et al. High-Parameter immune profiling with CyTOF. Methods Mol Biol 2020;2055:351-68.

17 Burch PA, Breen JK, Buckner JC, et al. Priming tissue-specific cellular immunity in a phase I trial of autologous dendritic cells for prostate cancer. Clin Cancer Res 2000;6:2175-82.

18 Sofi MH, Li W, Kaplan MH, et al. Elevated IL-6 expression in CD4 T cells via PKCtheta and NF-kappaB induces Th2 cytokine production. Mol Immunol 2009;46:1443-50.

19 Pedersen L, Idorn M, Olofsson GH, et al. Voluntary running suppresses tumor growth through epinephrine- and IL-6-dependent NK cell mobilization and redistribution. Cell Metab 2016;23:554-62.

20 Sartor O, Armstrong AJ, Ahaghotu C, et al. Survival of AfricanAmerican and Caucasian men after sipuleucel-T immunotherapy: outcomes from the proceed registry. Prostate Cancer Prostatic Dis 2020;23:517-26.

21 Pachynski RK. A "Tail" of Immunotherapy in Metastatic Prostate Cancer. Eur Urol 2020;78:831-3.

22 Antonarakis ES, Piulats JM, Gross-Goupil M, et al. Pembrolizumab for treatment-refractory metastatic castration-resistant prostate cancer: Multicohort, open-label phase II KEYNOTE-199 study. J Clin Oncol 2020;38:395-405.

23 Petrylak DP, Loriot Y, Shaffer DR, et al. Safety and clinical activity of Atezolizumab in patients with metastatic castration-resistant prostate cancer: a phase I study. Clin Cancer Res 2021;27:3360-9.

24 Gulley JL, Borre M, Vogelzang NJ, et al. Phase III trial of PROSTVAC in asymptomatic or minimally symptomatic metastatic castrationresistant prostate cancer. J Clin Oncol 2019;37:1051-61.

25 Sharma P, Pachynski RK, Narayan V, et al. Nivolumab plus ipilimumab for metastatic castration-resistant prostate cancer: preliminary analysis of patients in the CheckMate 650 trial. Cancer Cell 2020;38:489-99.

26 McNeel DG, Eickhoff JC, Wargowski E, et al. Concurrent, but not sequential, PD-1 blockade with a DNA vaccine elicits anti-tumor responses in patients with metastatic, castration-resistant prostate cancer. Oncotarget 2018;9:25586-96.

27 Stein MN, Fong L, Tutrone RF, et al. KEYNOTE-046: ADXS-PSA plus pembrolizumab (pembro) in metastatic castration-resistant prostate cancer (mCRPC). Journal of Clinical Oncology 2018;36:5019.

28 Redman JM, Steinberg SM, Gulley JL. Quick efficacy seeking trial (QuEST1): a novel combination immunotherapy study designed for rapid clinical signal assessment metastatic castration-resistant prostate cancer. J Immunother Cancer 2018;6:91.

29 Paller CJ, Olatoye D, Xie S, et al. The effect of the frequency and duration of PSA measurement on PSA doubling time calculations in men with biochemically recurrent prostate cancer. Prostate Cancer Prostatic Dis 2014;17:28-33. 